\title{
Toll-like receptor mRNA levels in alveolar macrophages after inhalation of endotoxin
}

\author{
N.A. Maris*,\#, M.C. Dessing*,\#, A.F. de Vos*,\#, P. Bresser ${ }^{\star}$, J.S. van der Zee`, \\ H.M. Jansen ${ }^{\circ}$, C.A. Spek ${ }^{\#}$ and T. van der Poll**\#
}

ABSTRACT: Toll-like receptors (TLRs) are pattern-recognition receptors that have been implicated in the initiation of innate immune responses upon the first encounter with invading pathogens. The airways are frequently exposed to various types of lipopolysaccharide (LPS) from the environment or from pathogens. The current study was designed to determine the effect of LPS on TLR gene expression in human alveolar macrophages in vivo.

In total, 16 healthy subjects were enrolled in a single-blinded, placebo-controlled study. Subjects inhaled $100 \mu \mathrm{g}$ LPS or normal saline ( $\mathrm{n}=\mathbf{8}$ per group). Measurements were performed in alveolar macrophages purified from bronchoalveolar lavage fluid obtained $6 \mathrm{~h}$ post-challenge.

Inhalation of LPS by healthy human volunteers resulted in enhanced alveolar macrophage expression of mRNAs encoding TLRs 1, 2, 7, 8 and CD14, and reduced expression of mRNAs encoding TLR4 and lymphocyte antigen 96.

In conclusion, lipopolysaccharide differentially influences the toll-like receptor mRNA expression profile in human alveolar macrophages in vivo.

KEYWORDS: Endotoxin, humans, lipopolysaccharide, lung, macrophages, toll-like receptors

$\mathbf{T}$ oll-like receptors (TLRs) are a family of cell-surface molecules involved in the recognition of structurally conserved pathogenassociated molecular patterns (PAMPs), such as lipopolysaccharide (LPS) [1, 2]. Thus far, 11 members of this family have been identified in the human genome. Most tissues or cells in healthy humans express at least one TLR, and several types express all (spleen, peripheral blood leukocytes) [3]. The expression profile of TLRs on individual cells is modulated by microbial products (e.g. LPS) and inflammatory mediators (e.g. tumour necrosis factor- $\alpha$ and interleukin-1 $\beta$ ), indicating that the expression of individual TLRs may alter during the course of infection, perhaps influencing the outcome of the immune response [1,2].

As the primary inflammatory cell found within the airways, alveolar macrophages play a central role in the constant recognition of potentially pathogenic organisms entering the lung [4]. Alveolar macrophages are equipped with different TLRs $[5,6]$. In murine alveolar macrophages, LPS has been shown to enhance the expression of TLR2 and myeloid differentiation primary response gene 88 mRNA, while reducing TLR4 and 5 mRNA levels [7]. Similarly, LPS increased TLR2 mRNA and protein levels in alveolar macrophages from healthy nonsmokers in vitro [6]. Knowledge of the regulation of alveolar macrophage TLR expression in the human lung in vivo is not available; this is an important issue in light of the constant exposure of the respiratory tract to LPS from the environment or foreign pathogens. The authors have quantitatively analysed TLR mRNA expression in alveolar macrophages harvested from human volunteers after inhalation of LPS.

\section{MATERIALS AND METHODS}

\section{Study subjects and design}

The study subjects were 16 nonsmoking males (mean \pm SD age $22.5 \pm 0.8$ yrs) recruited by advertising. Screening, consisting of a questionnaire, physical examination, routine blood and urine investigation, ECG and spirometry, did not reveal any abnormality. The study was approved by the Institutional Ethics and Research Committees, Academic Medical Center, University of Amsterdam, and written informed consent was obtained from all subjects before enrolment in the study. The subjects inhaled normal saline $(n=8)$ or LPS ( $\mathrm{n}=8$; from Escherichia coli O26:B6, $100 \mu \mathrm{g}$; Sigma-Aldrich, St Louis, MO, USA) using a largevolume reservoir delivery system as previously described [8,9]. LPS was purified by phenol extraction and had a protein content $<3 \%$; this specific LPS preparation has been used by the authors' and other laboratories in earlier studies with human volunteers [10-13]. Volunteers were randomised and blinded for the challenges they
AFFILIATIONS

${ }^{*}$ Center of Infection and Immunity

Amsterdam (CINIMA), and,

${ }^{*}$ Center for Experimental and

Molecular Medicine, and

'Dept of Pulmonology, Academic

Medical Center, University of

Amsterdam, The Netherlands.

CORRESPONDENCE

T. van der Poll

Academic Medical Center

Room G2-130

Meibergdreef 9

1105 AZ

Amsterdam

The Netherlands

Fax: 31206977192

E-mail: t.vanderpoll@amc.uva.nl

Received:

January 242006

Accepted after revision:

May 252006

SUPPORT STATEMENT

The study was supported by a grant (3.4.99.09) from the Dutch Asthma Foundation given to N.A. Maris. 
received. They were part of a larger study investigating the effects of inhaled salmeterol on lung inflammation induced by LPS, of which the results have been reported previously [11]. In this study, randomisation was balanced with two subjects per study day each from a different treatment group; the subjects analysed here represent the saline and LPS control groups that did not receive salmeterol. The analysis could not be carried out in one subject treated with LPS.

\section{Bronchoalveolar lavage and isolation of alveolar macrophages}

Bronchoalveolar lavage (BAL) was performed $6 \mathrm{~h}$ postchallenge in a standardised fashion according to the guidelines of the American Thoracic Society, using a flexible fibreoptic video bronchoscope. Eight successive $20 \mathrm{~mL}$ aliquots of prewarmed $0.9 \% \mathrm{NaCl}$ were instilled in a subsegment of the right middle lobe and each aspirated immediately with low suction. BAL fluid (BALF) was immediately centrifuged for $10 \mathrm{~min}$ at $250 \times g$ at $4{ }^{\circ} \mathrm{C}$. BALF cells were pushed through a $40 \mu \mathrm{m}$ nylon filter (BD Falcon, Bedford, MA, USA) and resuspended in icecold sterile automated magnetic cell sorting and separation (autoMACS) buffer (PBS, 0.5\% bovine serum albumin, $2 \mathrm{mM}$ EDTA; $\mathrm{pH}=7.4)$. Subsequently, cells were incubated for $15 \mathrm{~min}$ with CD71 microbeads (Multenyi Biotec, Bergisch Gladbach, Germany) at $4^{\circ} \mathrm{C}$ as previously described [11]. Cells were washed again in autoMACS buffer and purified by autoMACS (Multenyi Biotec). Total and viable cell counts were performed before and after the isolation procedure using a Burker-Turk haemocytometer and trypan blue (Emergo, Landsmeer, the Netherlands). Additionally, cytospins were prepared before and after autoMACS and stained with Giemsa. Total and differential cell counts revealed a purity and viability of isolated macrophages of $>95 \%$ in all groups.

\section{Multiplex ligation-dependent probe amplification}

After isolation, alveolar macrophages were dissolved in Trizol and stored at $-80^{\circ} \mathrm{C}$ until used for RNA isolation. RNA was isolated and analysed by multiplex ligation-dependent probe amplification as described [11, 14, 15] using a TLR-specific kit developed in collaboration with MRC-Holland (Amsterdam, the Netherlands) for the simultaneous detection of 17 mRNA molecules. Four different probe sets were generated for TLR4, corresponding with four different TLR4 splice variants (R01 to R04) identified in the National Center for Biotechnology Information (NCBI) database (www.ncbi.nlm.nih.gov); similarly, two probe sets for TLR8 were generated, corresponding with two different TLR8 splice variants (R01 and RO2; table 1). All samples were tested with the same batch of reagents. Levels of mRNA for each gene were expressed as a normalised ratio of the peak area divided by the peak area of the $\beta_{2}$ microglobulin mRNA (known to be unresponsive to LPS) [16], to give the relative abundance of mRNAs of the genes of interest $[11,14,15]$.

\section{Statistical analysis}

Values are expressed as mean \pm SE. Differences between groups were analysed by Mann-Whitney U-tests. A p-value of $<0.05$ was considered to represent a statistically significant difference.

\section{RESULTS}

When compared with inhalation of normal saline, inhalation of LPS modulated the expression of different TLR mRNAs in alveolar macrophages isolated from BALF $6 \mathrm{~h}$ post-challenge. LPS increased the expression of TLR1, 2, 7, 8 (R01) and LPS receptor (CD14) mRNAs in alveolar macrophages (fig. 1; all $\mathrm{p}<0.05$ versus saline). In contrast, the mRNA expression of TLR4 (R01 and R04) and lymphocyte antigen 96 (MD-2) decreased after inhalation of LPS (fig. 1 ; both $\mathrm{p}<0.05$ versus saline). TLR6 and TLR8 (R02) mRNA expression was not influenced by LPS challenge. TLR 3, 4 (R02), 5, 9 and 10 mRNAs were not detectable in alveolar macrophages obtained from either saline- or LPS-challenged volunteers.

\section{DISCUSSION}

From these data, it can be concluded that in alveolar macrophages, LPS reduces the levels of mRNAs regulating the expression of proteins involved in LPS signalling, in particular TLR4 and MD-2, whereas the levels of mRNAs regulating the expression of TLRs involved in the recognition of other PAMPs are either upregulated (TLR1, 2, 7 and 8(R01)) or not affected by LPS inhalation. It is worth noting that LPS did not significantly influence the expression of all four identified TLR4 splice variants. TLR4 (R01), of which the mRNA was downregulated after LPS inhalation, is involved in LPS binding and triggering of intracellular signal transduction cascades, while TLR4 (R03) and TLR4 (R04) lack residues 24-34, which are essential for MD-2 binding and LPS signalling [17]. LPS inhalation enhanced CD14 mRNA levels in alveolar macrophages; CD14 probably interacts with several PAMPs but is not directly involved in signal transduction.

The present results confirm and extend limited data obtained with murine and human alveolar macrophages in vitro. In line with the current results, stimulation with LPS in vitro has been shown to reduce TLR4 mRNA and increase TLR2 mRNA levels in murine alveolar macrophages [7] and to enhance TLR2 mRNA and protein levels in human alveolar macrophages [6]. TLR9 mRNA has not been detected in murine alveolar macrophages [18]. However, in contrast with the current human in vivo study, LPS did not influence the mRNA levels of TLR1, CD14 or MD-2 in murine alveolar macrophages in vitro [7]. Interestingly, corresponding with the present findings, intratracheal administration of LPS to mice in vivo reduced TLR4 mRNA levels in whole lungs and in recovered alveolar macrophages [19].

The current study has several limitations. First, it was singleblinded. Although unlikely, since only laboratory parameters were evaluated, this could have had an impact on the results. Secondly, only one time-point after lipopolysaccharide or saline inhalation was evaluated. Although a kinetic analysis involving mRNA harvesting at multiple time-points would be of considerable interest, the current authors chose not to expand the number of time-points in light of the invasive procedure to obtain bronchoalveolar lavage fluid samples and cells. This is also the reason why a relatively limited number of volunteers were studied. Thirdly, these findings are based on analysis of the relative abundance of mRNAs rather than on toll-like receptor protein expression. Nonetheless, the present study is the first to provide insight into the in vivo effect of lipopolysaccharide on toll-like receptor gene expression 
TABLE 1 Gene-specific oligonucleotides used for toll-like receptor (TLR) probe sets

Gene

Hybridising sequence of the small probe oligonucleotide Hybridising sequence of the long probe oligonucleotide
Reverse transcriptase primer sequence

CAAATGCGGCATCTTCA

TTTTGGAACACTAGACATTCCTA

TGATGACCCCCAAGAC

AGGAAAACCAATATAATGGAATC

TTGTCGGGGATTTTGTAGA

TTGTCGGGGATTTTGTAGA

TTGTCGGGGATTTTGTAGA

TTGTCGGGGATTTTGTAGA

ACCGGCCATGAGCAC

GTGGAACATTTTTCAAAAATACTA

GAGAGCAGAGCATGAGAG

GAACCAGATATTAGCAGGAA

GAACCAGATATTAGCAGGAA

CCTGCACCAGGAGAGAC

GTATTTCCAAGGATTTTACCACT

GGTCGCAGAGACGTG

CCTTAGAAAATTTTATTCCCTTG

MD-2

AGCGCGCGTCCTGCTTGTTGCTGCTGCTGCTGCCGCTGGTCATG GGGTTCCCTAAGGGTTGGAGAGGTTCGGAAGACTTATCGACCATGG

AGGGAGAGACTGTGAATACAACAATATCATTCTCCTTCAAGGGCATG GGGTTCCCTAAGGGTTGGACTGATGACGATTACTCTTTTTGCAGAGCTCTGA

B2M: $\beta_{2}$ microglobulin; R01, 02, 03 and 04: indicate splice variants; D01: indicates that probe also reacts with contaminating genomic DNA; CD14: lipopolysaccharide receptor; MD-2: lymphocyte antigen 96. In order to avoid detection of contaminating DNA fragments, all target sequences (except TLR6) have an exon boundary close to the probe ligation site. Some TLRs (4 and 8) are represented by different splice variants which have been identified before. TLR4 variant 1 is involved in lipopolysaccharide (LPS) binding and triggering of intracellular signal transduction cascades, while variants 3 and 4 lack residues 24-34 which are essential for MD-2 binding and LPS signalling [17]. The biological roles of TLR4 variant 2 and TLR8 variants remain to be elucidated. 

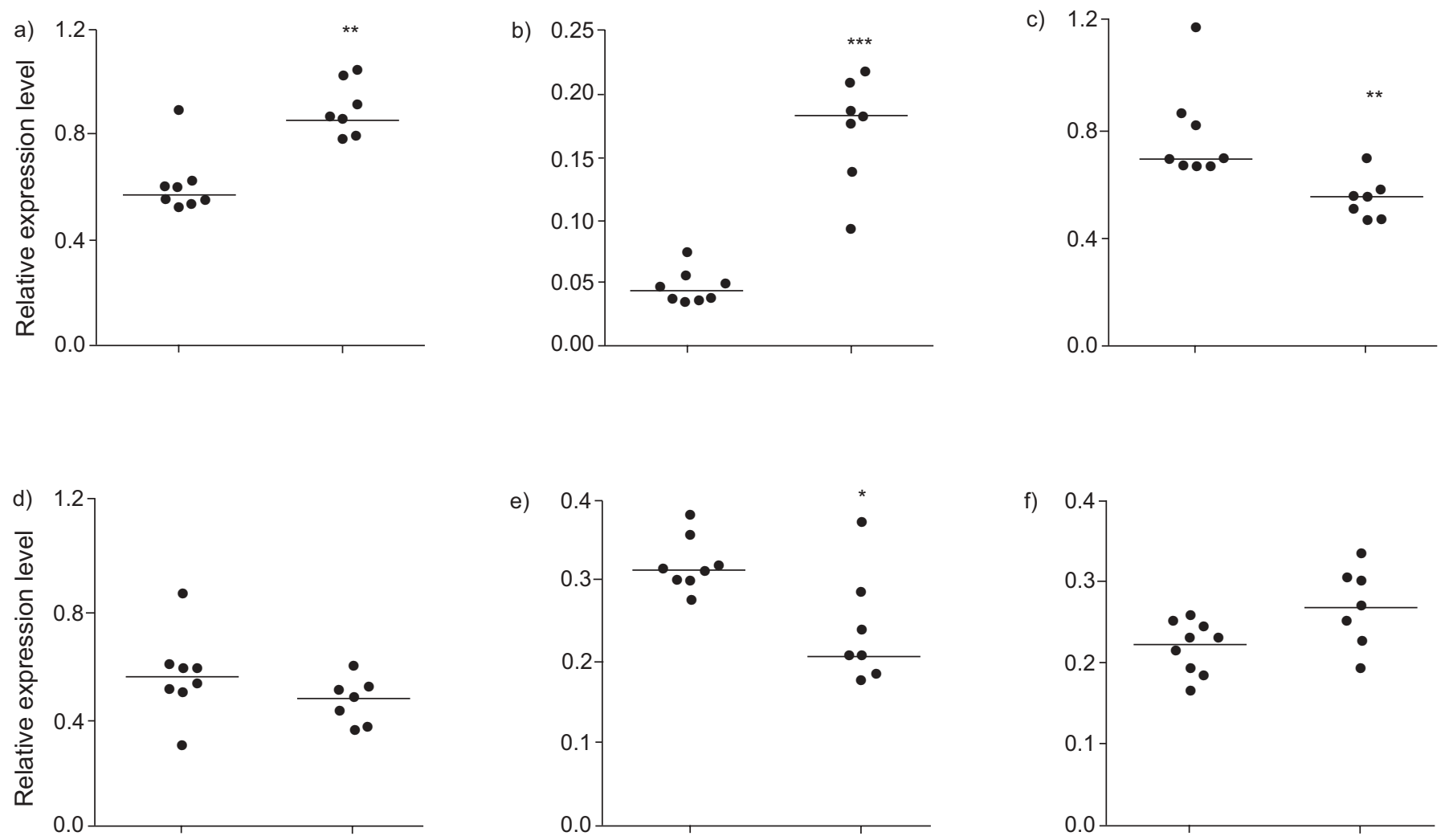

e)
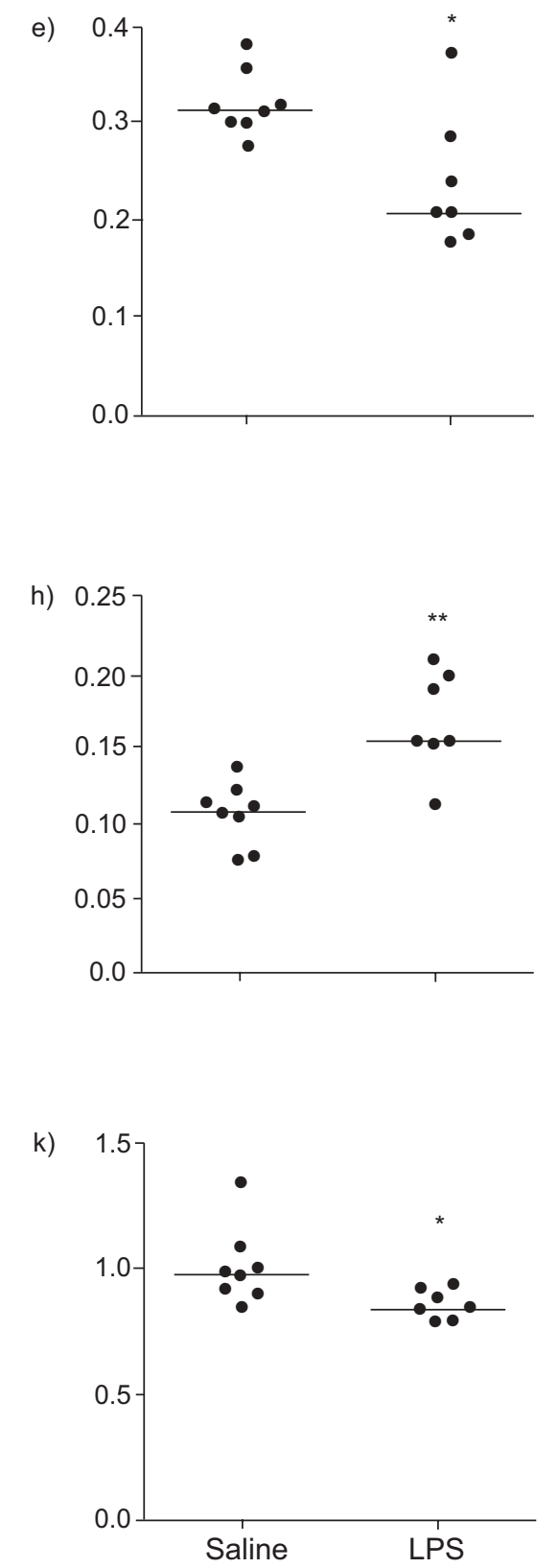
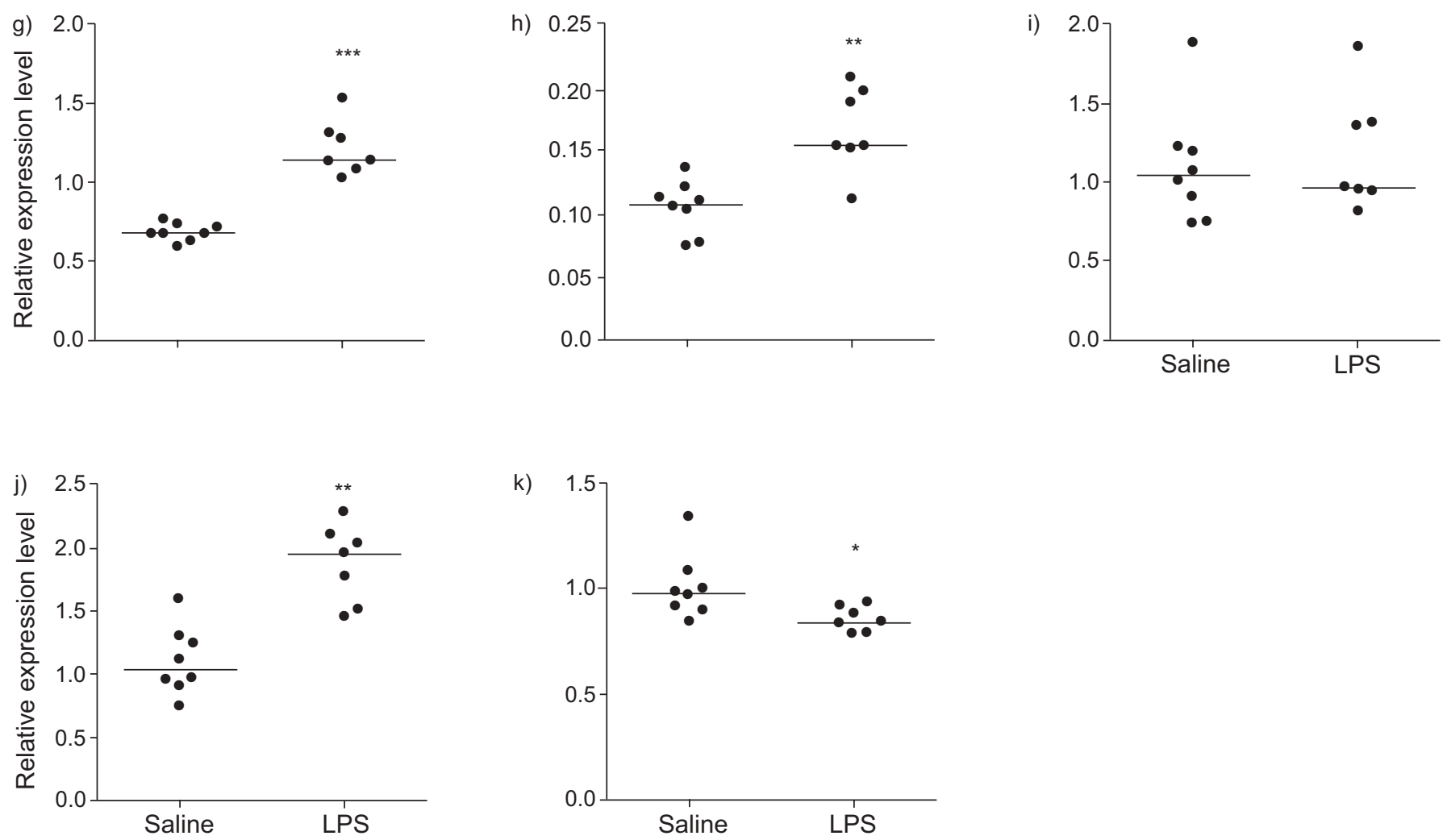

FIGURE 1. Relative expression of alveolar macrophage toll-like receptor (TLR) mRNAs after exposure to lipopolysaccharide (LPS) in: a)TLR1; b) TLR2; c)TLR4 (splice variant R0)1; d) TLR4 R03; e) TLR4 R04; f) TLR6; g) TLR7; h) TLR8 R01; i) TLR8 R02; j) CD14; and k) lymphocyte antigen 96. Data represent levels of mRNA species relative to $\beta_{2}$ microglobulin in each individual. Horizontal lines represent medians. *: $p<0.05$ versus saline; **: $p<0.01$ versus saline; $* * *: p<0.001$ versus saline (Mann-Whitney U-test). 
profiles in human lungs. Further studies are warranted to evaluate the functional consequences of these lipopolysaccharide effects in alveolar macrophages.

\section{REFERENCES}

1 Takeda K, Akira S. Toll-like receptors in innate immunity. Int Immunol 2005; 17: 1-14.

2 Kawai T, Akira S. Pathogen recognition with toll-like receptors. Curr Opin Immunol 2005; 17: 338-344.

3 Zarember KA, Godowski PJ. Tissue expression of human toll-like receptors and differential regulation of toll-like receptor mRNAs in leukocytes in response to microbes, their products, and cytokines. J Immunol 2002; 168: 554-561.

4 Twigg HL 3rd. Macrophages in innate and acquired immunity. Semin Respir Crit Care Med 2004; 25: 21-31.

5 Droemann D, Goldmann T, Branscheid D, et al. Toll-like receptor 2 is expressed by alveolar epithelial cells type II and macrophages in the human lung. Histochem Cell Biol 2003; 119: 103-108.

6 Droemann D, Goldmann T, Tiedje T, Zabel P, Dalhoff K, Schaaf B. Toll-like receptor 2 expression is decreased on alveolar macrophages in cigarette smokers and COPD patients. Respir Res 2005; 6: 68.

7 Oshikawa K, Sugiyama Y. Gene expression of Toll-like receptors and associated molecules induced by inflammatory stimuli in the primary alveolar macrophage. Biochem Biophys Res Commun 2003; 305: 649-655.

8 van der Veen MJ, van der Zee JS. Aerosol recovery from large-volume reservoir delivery systems is highly dependent on the static properties of the reservoir. Eur Respir J 1999; 13: 668-672.

9 van der Veen MJ, Van Neerven RJ, De Jong EC, Aalberse RC, Jansen HM, van der Zee JS. The late asthmatic response is associated with baseline allergenspecific proliferative responsiveness of peripheral $\mathrm{T}$ lymphocytes in vitro and serum interleukin-5. Clin Exp Allergy 1999; 29: 217-227.

10 Maris NA, de Vos AF, Bresser P, et al. Activation of coagulation and inhibition of fibrinolysis in the lung after inhalation of lipopolysaccharide by healthy volunteers. Thromb Haemost 2005; 93: 1036-1040.

11 Maris NA, de Vos AF, Dessing MC, et al. Antiinflammatory effects of salmeterol after inhalation of lipopolysaccharide by healthy volunteers. Am J Respir Crit Care Med 2005; 172: 878-884.

12 Sandstrom T, Bjermer L, Rylander R. Lipopolysaccharide (LPS) inhalation in healthy subjects increases neutrophils, lymphocytes and fibronectin levels in bronchoalveolar lavage fluid. Eur Respir J 1992; 5: 992-996.

13 Michel O, Dentener M, Corazza F, Buurman W, Rylander R. Healthy subjects express differences in clinical responses to inhaled lipopolysaccharide that are related with inflammation and with atopy. J Allergy Clin Immunol 2001; 107: 797-804.

14 Spek CA, Verbon A, Aberson H, et al. Treatment with an anti-CD14 monoclonal antibody delays and inhibits lipopolysaccharide-induced gene expression in humans in vivo. J Clin Immunol 2003; 23: 132-140.

15 Wettinger SB, Doggen CJ, Spek CA, Rosendaal FR, Reitsma PH. High throughput mRNA profiling highlights associations between myocardial infarction and aberrant expression of inflammatory molecules in blood cells. Blood 2005; 105: 2000-2006.

16 Suzuki T, Hashimoto S, Toyoda N, et al. Comprehensive gene expression profile of LPS-stimulated human monocytes by SAGE. Blood 2000; 96: 2584-2591.

17 Nishitani C, Mitsuzawa H, Hyakushima N, Sano H, Matsushima N, Kuroki Y. The toll-like receptor 4 region Glu24-Pro34 is critical for interaction with MD-2. Biochem Biophys Res Commun 2005; 328: 586-590.

18 Suzuki K, Suda T, Naito T, Ide K, Chida K, Nakamura H. Impaired toll-like receptor 9 expression in alveolar macrophages with no sensitivity to CpG DNA. Am J Respir Crit Care Med 2005; 171: 707-713.

19 Fan J, Kapus A, Marsden PA, et al. Regulation of toll-like receptor 4 expression in the lung following hemorrhagic shock and lipopolysaccharide. J Immunol 2002; 168: 5252-5259. 